\title{
Assessment on the applicability of finite difference methods to model light propagation in photonic liquid crystal fibers
}

\author{
Katarzyna A. Rutkowska, ${ }^{* 1}$ Li-Wei Wei ${ }^{2}$ \\ ${ }^{I}$ Faculty of Physics, Warsaw University of Technology, Koszykowa 75, PL-00662 Warsaw, Poland \\ ${ }^{2}$ Institute of Micromechanics and Photonics, Warsaw Univ. of Technology, Boboli 8, PL-02525 Warsaw, Poland
}

Received December 27, 2012; accepted December 28, 2012; published December 31, 2012

\begin{abstract}
In this report we show our numerical scheme based on a finite difference approach and devoted to modeling light propagation in photonic liquid crystal fibers. Our method allows for optical anisotropy of liquid crystals to be included in calculations. In particular, a vectorfield mode solver implemented here allows considering an arbitrary rotation of LC molecules in the transverse plane with respect to the propagation axis. To this end, suitable analytical formulations have been derived and numerically implemented. The computational scheme developed has been optimized and checked for numerical convergence. The achieved results have been validated in accordance to those described in literature or obtained from commercial software. A qualitative comparison with the experimental data obtained for a particular sample of PLCF has been also made.
\end{abstract}

Photonic crystal fibers (PCFs) are made of 2D photonic crystals extended homogeneously over the propagation direction [1]. The cross-section of a specific PCF (fabricated at the Maria Curie-Sklodowska University in Lublin, Poland), consisting of three coaxial rings of airholes distributed in the silica cladding, is shown in Fig. 1a. In the PCF presented here the core is made of a solid substance but in some designs it may remain hollow. By changing: (i) the geometry of a $2 \mathrm{D}$ periodic structure (e.g. the size, shape and location of air-holes) and (ii) the parameters (e.g. the size and material(s) out of which the core is made) it is possible to adjust guiding properties of PCFs. Therefore the photonic bandgap (PBG) guiding or/and the index guiding (based on the modified total internal reflection, mTIR) can be (both) present in PCF structures. It has been shown that infiltration of the airholes (e.g. with gaseous and liquid materials) may lead to even more extraordinary properties of PCFs. In particular, the application of liquid crystals (LCs) has resulted in a new type of photonic structures typically referred to as photonic liquid crystal fibers (PLCFs). High tunability of PLCFs (manifested by, inter alia, shifting in the spectral position of the bandgaps and/or switching between the guiding mechanisms) [2] arises from special optical properties of LCs [3] which can be dynamically adjusted by: (i) external fields and factors (including electric and magnetic fields, temperature, strain and pressure) and (ii) an optical beam itself (i.e. when nonlinear effects take place in LCs). The chromatic dispersion for a typical

\footnotetext{
*E-mail: kasia@if.pw.edu.pl
}

nematic liquid crystal (NLC) to be used as infiltration is presented in Fig. 1b [4]. Due to the fact that both ordinary and extraordinary refractive index of 6CHBT NLC are higher than that of the silica glass, PBG guiding is present in the PLCF under consideration, while an mTIR-based guiding mechanism is characteristic for the empty PCF of interest (see Figs. 3-4). Moreover, when molecular orientation and optical anisotropy of LCs are taken into account a more complex scenario for light propagation in PLCF is obtained. In particular, modal degeneracy upon polarization and modal birefringence, as well as single polarization guidance can be observed for a particular orientation of an LC within a specific spectral range.

In this paper we present our numerical approach developed to model light guidance in PLCFs with a possibility of optical anisotropy of LCs to be implemented in calculations. The numerical convergence of computational schemes and correctness of obtained results have been checked and discussed. Quantitative and qualitative comparisons with results: (i) described in literature or obtained with use of commercial software and (ii) obtained in experimental conditions, have been performed, respectively.
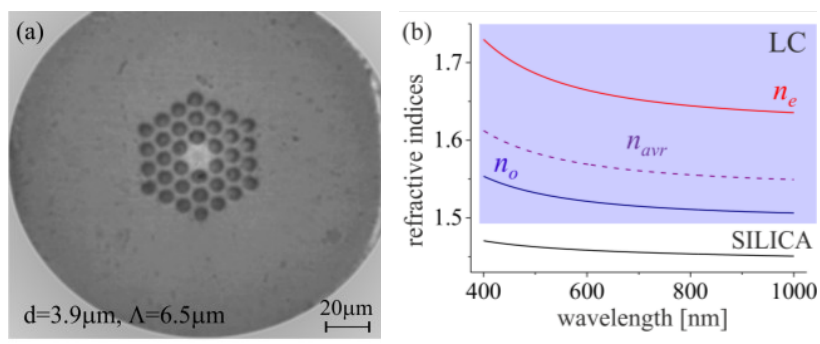

Fig. 1. (a) Photo of the host PCF, where $d$ is the diameter of the airholes and $\Lambda$ is the pitch. (b) Refractive indices of 6CHBT NLC as a function of wavelength [4], where $n_{o}$ is the ordinary and $n_{e}$ is the extraordinary refractive index; its average value specified for a randomly oriented NLC is defined as: $n_{a v r}=2 n_{o} / 3+\mathrm{n}_{\mathrm{e}} / 3$ (dashed line). The refractive index of silica is also shown for comparison (black line).

The numerical scheme presented here is based on the vector-field FDFD mode solver described in [5] with the Yee's staggered mesh applied (to reduce the number of grid points while maintaining high accuracy). Specifically, by introducing some correction terms (marked in red in Eqs. 5a-d), we have been able to extend the original 
FDFD scheme proposed in [5] in such a way that apart from diagonal electric anisotropy it is capable of accounting transverse material anisotropy in our calculations. It means that for the PLCF analyzed here, an arbitrary rotation of LC molecules in the transverse plane with respect to the fiber axis can be considered. Moreover, our scheme gives effective refractive indices for two orthogonal polarizations and thus reports on the birefringence and polarization mode dispersion of the specific structure. It also allows for identification of the hybrid-guiding, which can be obtained for some configurations of PCF when index-guiding is observed for one and PBG-guiding for the other state of polarization. Specifically, the transverse components of electric and magnetic fields can be found by solving the eigenvalue problems defined by Eqs. (1)-(2), while corresponding longitudinal components are calculated with use of Eqs. (3)-(4):

$$
\begin{aligned}
& {\left[\begin{array}{ll}
P_{x x} & P_{x y} \\
P_{y x} & P_{y y}
\end{array}\right]\left[\begin{array}{l}
E_{x} \\
E_{y}
\end{array}\right]=\beta^{2}\left[\begin{array}{l}
E_{x} \\
E_{y}
\end{array}\right],} \\
& {\left[\begin{array}{ll}
Q_{x x} & Q_{x y} \\
Q_{y x} & Q_{y y}
\end{array}\right]\left[\begin{array}{l}
H_{x} \\
H_{y}
\end{array}\right]=\beta^{2}\left[\begin{array}{l}
H_{x} \\
H_{y}
\end{array}\right],} \\
& i k_{0} \varepsilon_{z z} E_{z}=V_{y} H_{x}-V_{x} H_{y}, \quad i k_{0} H_{z}=U_{x} E_{y}-U_{y} E_{x},
\end{aligned}
$$

where $\beta=k_{0} n_{\text {eff }}$ is the propagation constant, $k_{0}$ is a wavevector, $n_{\text {eff }}$ are effective refractive indices of the modes, and $P, Q$ are the global matrices for the electric and magnetic field, respectively, with the sub-matrices defined as follows:

$$
\begin{aligned}
P_{i i} & =U_{i}\left(\frac{I}{\varepsilon_{z z}}\right)\left[V_{j} \varepsilon_{j i}-\frac{1}{k_{0}^{2}} V_{j} V_{i} U_{j}\right]+ \\
& +\left[k_{0}^{2} I+U_{i}\left(\frac{I}{\varepsilon_{z z}}\right) V_{i}\right]\left[\varepsilon_{i i}+\frac{1}{k_{0}^{2}} V_{j} U_{j}\right], \\
P_{i j}= & U_{i}\left(\frac{I}{\varepsilon_{z z}}\right) V_{j}\left[\varepsilon_{i i}+\frac{1}{k_{0}^{2}} V_{i} U_{i}\right]+U_{i}\left(\frac{I}{\varepsilon_{z z}}\right) V_{i} \varepsilon_{i j}+ \\
& +k_{0}^{2} \varepsilon_{i j}-\frac{1}{k_{0}^{2}}\left[k_{0}^{2} I+U_{i}\left(\frac{I}{\varepsilon_{z z}}\right) V_{i}\right] V_{j} U_{i}, \\
Q_{i i}= & -\frac{1}{k_{0}^{2}} V_{i} U_{j} U_{i}\left(\frac{I}{\varepsilon_{z z}}\right) V_{j}+\varepsilon_{j i} U_{i}\left(\frac{I}{\varepsilon_{z z}}\right) V_{j}+ \\
& +\left[\varepsilon_{j j}+\frac{1}{k_{0}^{2}} V_{i} U_{i}\right]\left[k_{0}^{2} I+U_{j}\left(\frac{I}{\varepsilon_{z z}}\right) V_{j}\right], \\
Q_{i j}= & -\left[\varepsilon_{i j}+\frac{1}{k_{0}^{2}} V_{i} U_{i}\right] U_{j}\left(\frac{I}{\varepsilon_{z z}}\right) V_{i}-k_{0}^{2} \varepsilon_{j i}+ \\
& -\varepsilon_{j i} U_{i}\left(\frac{I}{\varepsilon_{z z}}\right) V_{i}+\frac{1}{k_{0}^{2}} V_{i} U_{j}\left[k_{0}^{2} I+U_{i}\left(\frac{I}{\varepsilon_{z z}}\right) V_{i}\right],
\end{aligned}
$$

where $i \equiv(x, y) ; j \equiv(x, y)$ and $i \neq j$. Please note that when $\varepsilon_{i j}=0$, the sub-matrices in Eqs. (5a-d) reduce to those in [5]. The finite-difference (FD) matrices (with an assumption of the Dirichlet boundary conditions) are given by:

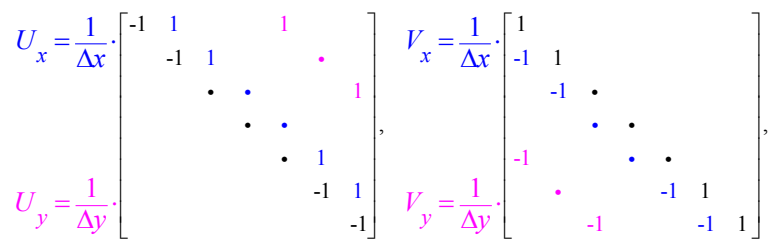

where non-zero values of a particular FD matrix are marked with a specific color for off- and with black for on-diagonal elements, respectively.

It is worth noting that in this work an index-averaging technique has been implemented in order to improve the performance of the FDFD scheme.

First, in order to verify the correctness of the FDFD scheme proposed, numerical tests for various fiber geometries have been performed and their results compared with proper references. In particular, a holey PCF, which represents the same type of geometry as the PCF of interest, has been tested. This specific structure is sketched in Fig. 2a. Convergences curves (@ $\lambda=1.5 \mu \mathrm{m})$ for the $x$-polarized fundamental (core) mode (shown in Fig. 2b) are presented in Fig. 2c.

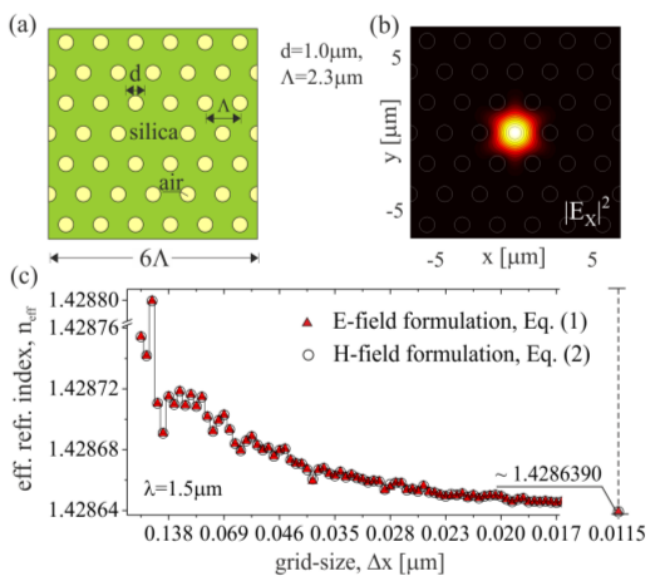

Fig. 2. (a) Scheme and geometrical parameters of the periodic photonic structure in the form of a holey fiber. (b) Intensity spatial profile (represented here by $\left|E_{x}\right|^{2}$ ) for the fundamental (core) mode. (c) Convergence curves for the fundamental mode obtained with use of vector-field FDFD scheme for $E$ - and $H$-field formulation, respectively. Full correspondence between them (also within whole spectral range) is observed.

The simulations performed have confirmed that the fundamental modes for two orthogonal polarizations are degenerated (i.e. are characterized by the same effective indices). Such degeneracy is characteristic for triangularlattice PCFs [6]. For the holey fiber geometry from Fig. 2, the results of our vector-field FDFD scheme differs by around $4 \cdot 10^{-5}(\mathrm{RE}=28 \mathrm{ppm})$ from that given in [5].

Since in this work we put an emphasis on PLCFs, our numerical tool has been also tested with regards to the PCF sample (Fig. 1a) infiltrated with a typical NLC (6CHBT, Fig. 1b). Transmission spectra measured in experimental conditions for a PCF before and after infiltration are shown in Fig. 3. As one can see, it 
continues for an empty PCF, while several transmission peaks within the spectral range of the light source exist for the PLCF sample (due to the PBG guiding).

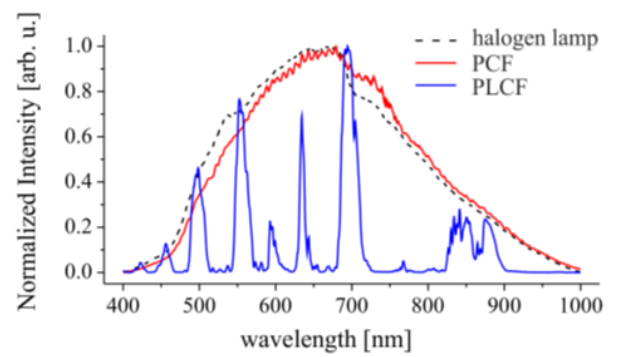

Fig. 3. Experimental results showing normalized transmission spectra for a 40-cm-long PCF (red) and for its $5-\mathrm{cm}$ section infiltrated with 6CHBT NLC (blue). Dash line represents the normalized spectrum of a light source (halogen lamp).

The above observations can be confirmed numerically when suitable simulations are performed. In fact, effective refractive indices of the fundamental core mode with respect to the wavelength for PCF and PLCF are shown in Fig. 4. However, owning to the limit of experimental means, direct quantitative comparison is not quite accessible. Instead, qualitative evaluation of the experimental and theoretical results may be performed.

For a PCF the first two modes (with the highest effective indices) are degenerated upon polarization. It has been also confirmed by analogous simulations performed with the use of commercial software based on finite elements methods (Comsol, Multiphysics), which are both quantitatively and qualitatively consistent.

For the PLCF sample, the isotropic form of the permittivity tensor for an LC (with values of $n_{\text {avr }}{ }^{2}$ on its diagonal) has been used as a first approximation of zero orientation order of an LC within a PCF.

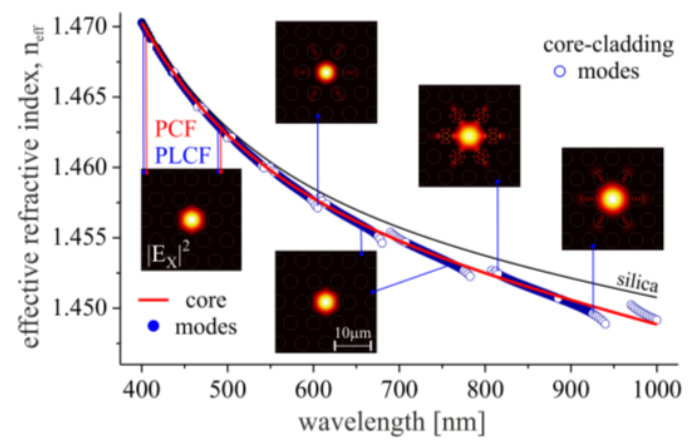

Fig. 4. Numerical results on the effective refractive index of the fundamental (quasi- $x$-polarized) core mode in the PCF (red line) and PLCF sample (blue dots). Hollow circles indicate the cases in which modal field extends significantly beyond the core region (light is guided not only in the core but also in the silica cladding and/or in LC) - in some of them imaginary part of an effective index occurs. Insets show $\left|E_{x}\right|^{2}$, giving thus information about the intensity pattern for some specific wavelengths.

It is worth to mention that PBG-guiding modes have very different field patterns when compared to those of the empty PCF. Specifically, in addition to the core-guiding modes and typical cladding modes (when light propagates in silica), some LC-guiding modes are also observed. Moreover, unlike the modes in a PCF, the modal patterns in the PBG-guiding PLCF sample change significantly with the wavelength, and for some of them, core-guiding modes are not present. The results obtained for the PLCF suggest that, in general, two orthogonal polarizations are not degenerated (which was confirmed by analogous simulations performed with the use of the Comsol software).

It is important to note that in the analyzed case of the PLCF structure, it is possible to align and/or realign the LC molecules within the fiber when proper orientation treatment is performed and/or external fields are applied [2]. While our FDFD scheme allows transverse anisotropy to be accounted, it is possible to study the effects of LC reorientation (in the transverse plane) on the propagation properties of the PLCF. Exemplary results of simulations performed for different orientational angle $\phi$ (between long axis of LC molecules and fiber axis) are presented below:

\begin{tabular}{|c|c|c|c|c|c|c|c|c|}
\hline & \multirow{2}{*}{$\begin{array}{l}\text { empty } \\
\text { PCF }\end{array}$} & \multicolumn{7}{|c|}{$\phi / \pi$} \\
\hline & & 0 & $1 / 12$ & $1 / 6$ & $1 / 4$ & $1 / 3$ & $5 / 12$ & $1 / 2$ \\
\hline$\lambda=650 \mathrm{~nm}$ & \multirow{3}{*}{ DG } & MB & $\mathrm{CCM}$ & & \multirow{3}{*}{ DG } & & $\mathrm{NCM}$ & MB \\
\hline$\lambda=775 \mathrm{~nm}$ & & MB & MB & & & & MB & MB \\
\hline$\lambda=800 \mathrm{~nm}$ & & $\mathrm{NCM}$ & MB & & & & $\mathrm{NCM}$ & SP \\
\hline
\end{tabular}

As one can see, it is possible to find wavelengths for which continuous molecular reorientation is possible with a fundamental core mode always guided by the structure. For some specific settings (wavelength or/and molecular orientation) core-guided mode: (i) is birefringent (MB), (ii) is degenerated (DG), (iii) is dominated by the corecladding mode (CMM), (iv) appears only for single polarization (SP), or (v) does not propagate in the structure (NCM). For $\phi=\pi / 6, \pi / 4, \pi / 3$ analyzed structure behaves like an empty one (i.e., no gaps are present and the fundamental modes are degenerated upon polarization). Future examinations on both the experiment and the numerical schemes are required to justify the simulations.

The authors acknowledge Homing Plus Programme granted by the Foundation for Polish Science (HOMING PLUS/2010-2/11) and co-financed by the European Regional Development Fund.

\section{References}

[1] P. St. J. Russell, Science 299, 358 (2003).

[2] T.R. Woliński et al., Phot. Lett. Poland 3(1), 20 (2011).

[3] I-C. Khoo, S-T. Wu, Optics and Nonlinear Optics of Liquid Crystals (World Scientific Pub., Singapore 1993).

[4] J. Schirmer et al., Mol. Cryst. Liq. Cryst. 307(1), 17 (1997).

[5] Z. Zhu, T.G. Brown, Opt. Exp. 10(17), 853 (2002).

[6] M. Koshiba, K. Saitoh, IEEE Phot. Techn. Lett. 13(12), 1313 (2001). 